\title{
Thermal And Thermo-Mechanical Modelling of Polymer Overmoulded Electronics
}

\author{
F. Sarvar ${ }^{1}$, D. C. Whalley ${ }^{1}$, D. A. Hutt ${ }^{1}$, N.J. Teh ${ }^{2}$ and P.J. Palmer ${ }^{1}$ \\ ${ }^{1}$ Wolfson School of Mechanical and Manufacturing Engineering \\ Loughborough University, Loughborough, Leics, LE11 3TU, UK \\ ${ }^{2}$ TWI, Granta Park, Great Abington, Cambridge, CB1 6AL
}

\begin{abstract}
1. ABSTRACT
This paper reports on the results from a research project investigating a novel technology for the manufacture of recyclable polymeric modules with embedded electronic controls and power distribution. The aim of this project is to develop a technology that fully encapsulates electronics for use in the demanding automotive environment. A two shot moulding technology will protect delicate electronic circuitry mounted outside of the passenger compartment from extremes of temperature, vibration and humidity. The resultant assemblies will also be readily recyclable, making it possible to cost-effectively separate electronic components from the polymer at the end of vehicle life, allowing the recovery of high purity recyclate.
\end{abstract}

The encapsulating polymers will have low thermal conductivity, so the process of encapsulation will introduce a thermally insulating barrier around the electronics, which will impact on the dissipation of heat from the components. In addition, the thermal performance of the assembly will be further affected by the high temperature environments within which some of these electronic modules will have to operate, such as under the bonnet of a vehicle.

This paper will present the results of preliminary models developed for investigating the thermal and mechanical issues arising during the operation of such encapsulated electronics. Analytical models and finite element techniques have been employed to simulate the thermo-mechanical behaviour of overmoulded printed circuit boards.

\section{INTRODUCTION}

The encapsulation of electronics within polymer materials is of great interest to the automotive industry as a means to protect components and circuitry operating in harsh environments, thereby enabling them to be utilised in ever more demanding areas of the vehicle. Furthermore, with the drive to reduce vehicle weight, the use of overmoulding with thermoplastic polymers will enable the embedding of electronic functionality in structural/mechanical components of the vehicle thereby saving space and weight. However, there are increasing legislative demands to recycle vehicles at the end-of-life and, under the EoLV directive [1], it is the producer's responsibility to ensure that vehicles are designed and manufactured in such a way as to allow reuse, recycling and recovery to be achieved. It is therefore important that any new technology employed does not compromise its cost effective disassembly. Within the context of achieving recyclable polymer encapsulated electronic modules, one of the major challenges is the clean separation of original sub-assemblies from the overmoulding polymer, which is injected at high temperatures and/or pressures and will have filled all the gaps between adjacent components and adhered to the various surfaces of the PCB-based circuitry. In order to overcome this problem, the use of water-soluble polymers has been investigated as a means to separate the electronics at the end of life. Initially, these are used to overmould the electronics in a first moulding step. However, in order to prevent dissolution of these water soluble polymers in the harsh operating environments, a two-shot moulding technique is used to apply a second overmoulding layer of an engineering thermoplastic polymer that provides protection and can form a structural element of one of the vehicles components, such as a bumper or a motor housing. At the end of life, by exposing and dissolving the inner water soluble polymer, the electronics can be efficiently separated.

The overmoulding of electronic circuits with a low thermal conductivity polymer will affect their thermal performance. For devices dissipating significant power this could result in significant overheating and premature failure of the electronic components, or to thermal distortion/damage to the structural polymer part containing the electronics. In addition, the differences in the coefficient of thermal expansion (CTE) between the electronic components and encapsulant could cause undue stresses within the module that may result in failure of solder joints and substrate copper tracks.

The work presented here is part of a major collaboration between a number of companies and research establishments. A detailed investigation was 
carried out by the project partners to identify a suitable polymer formulation for the first overmould shot. In addition to solubility the main criteria were increased thermal conductivity and good adhesion with the electronic components to improve thermal performance and ensure maximum heat transfer across the board/polymer interface. Techniques were also developed to reduce the injection pressure during the overmoulding process in order to limit the induced stresses and distortions that might occur to the electronics.

In order to understand the effect of the polymer encapsulation on thermo-mechanical performance, simulations and experimental studies were conducted. Both analytical and finite element methods have been used and within this paper, results will be presented of the models developed to establish the effect that the polymer thermal conductivity, thermal expansion and mechanical properties have on the performance of the encapsulated device. Simulation studies have been conducted using the candidate polymer to ascertain the de-rating level required to achieve similar temperatures to that of the circuit without any overmoulding. In addition to material properties variation, the effect of the polymer structure has been investigated using $2 \mathrm{D}$ axisymmetric models that have demonstrated that voids in the polymer adjacent to a heat source, such as a resistor, can cause a significant increase in the temperature rise at that location.

\section{MODELLING TECHNIQUES}

Several techniques were used to model the thermal performance of the device and to produce results that could be validated by comparison with experimental structures. Simple analytical one-dimensional conduction models and 2D axisymmetric finite element (FE) modelling were used first and then full 3D FE modelling techniques were employed for more detailed analysis.

The work described in this paper will mainly focus on a simple encapsulated test circuit, as shown in figure 1, which comprises of three surface mounted chip resistors, acting as heat sources, and which are soldered onto an FR4 substrate and then overmoulded with a layer of recyclable, water soluble polymer and a further layer of an engineering polymer, 30\% glass filled (GF) polybutylene terephthalate (PBT).

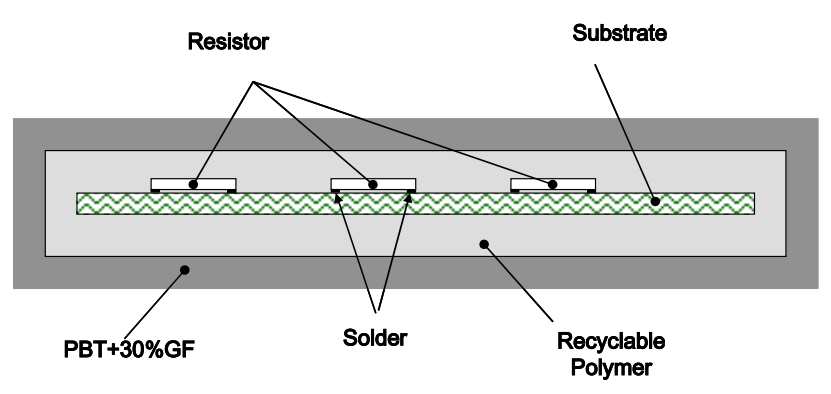

Fig. 1 Test structure
The Unigraphics Solutions EDS I-DEAS Master Series NX finite-element package was used for pre- and post- processing of all of the FE thermal models. This was coupled with the Maya Heat Transfer Technologies' TMG (Thermal Model Generator) FD solver to simulate the heat transfer within the modules.

The modelling techniques used here have been described and validated in full in a previous publication [2]. Here these techniques will be used to identify the effects of overmoulding on thermal and thermomechanical performance of simple assemblies.

For modelling of the thermo-mechanical effects of the predicted temperatures, the results from the thermal solver are used as boundary conditions in a second, solid mechanics, analysis using the ABAQUS FE solver. IDEAS ABAQUS Data Translator was used to define solution parameters for ABAQUS. The translator provides bi-directional exchange of FE models and simulation results with ABAQUS solvers. FE models constructed in I-DEAS can be directly written to an ABAQUS input file and ABAQUS results can be directly imported back into I-DEAS for post-processing

\subsection{Material Properties}

It is clear that any predicted result can only be as accurate as the material properties used. Since the thermomechanical properties for the novel water soluble polymer blend used in overmoulding the chip/substrate combinations were not available, experimental property measurements were carried out using TMA, DMA and DSC techniques.

The properties used in the simulations were for alumina chip resistors, FR4 substrate, a typical glass filled PBT polymer and alumina filled water soluble polymer (WSP) as given in Table 1 . The elastic modulus for the recyclable polymer was measured as a function of temperature as shown in figure 2 ; values are given at two temperatures in the table.

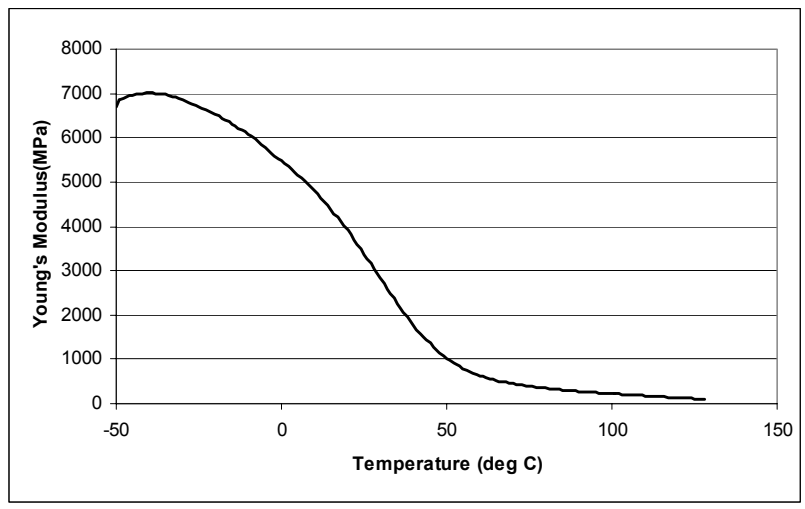

Fig. 2 Measured elastic modulus for the recyclable, water soluble polymer 


\begin{tabular}{|c|c|c|c|c|}
\hline Material & $\begin{array}{c}\text { Elastic } \\
\text { Modulus GPa }\end{array}$ & $\begin{array}{c}\text { Density } \\
\mathrm{kg} / \mathrm{m}^{3}\end{array}$ & $\begin{array}{c}\text { Thermal } \\
\text { Conductivity } \\
\mathrm{W} / \mathrm{mK}\end{array}$ & $\begin{array}{c}\mathrm{CTE} \\
\mu \mathrm{m} /{ }^{\circ} \mathrm{C}\end{array}$ \\
\hline Alumina & 370 & 3890 & 25 & 8.2 \\
\hline $\mathrm{FR} 4$ & 20.6 & 1900 & 0.3 & 11.7 \\
\hline $\begin{array}{c}\mathrm{WSP}+ \\
30 \% \mathrm{Al}_{2} \mathrm{O}_{3}\end{array}$ & $\begin{array}{c}3.9 @ 20^{\circ} \mathrm{C} \\
\mathrm{0} \% \mathrm{G} 100^{\circ} \mathrm{C}\end{array}$ & 1520 & 0.43 & 170 \\
\hline $\begin{array}{c}30 \% \mathrm{GF} \\
\mathrm{PBT}\end{array}$ & 9.97 & 1527 & 0.22 & 77.7 \\
\hline
\end{tabular}

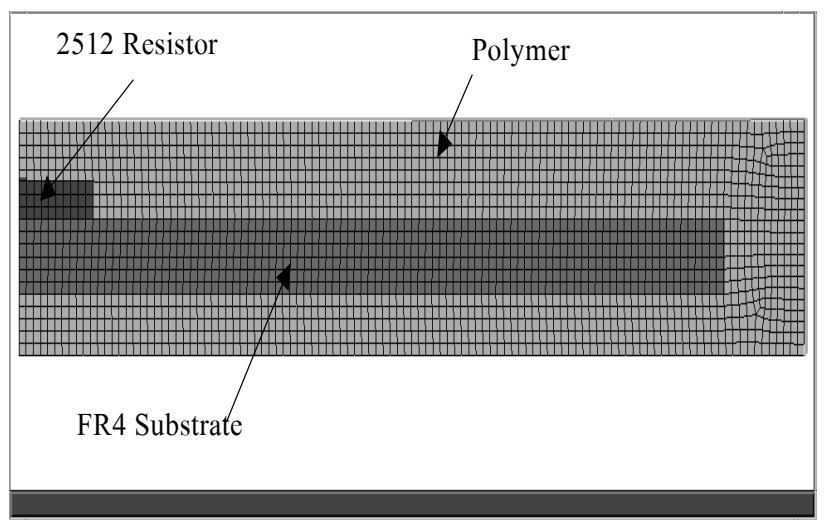

Fig. 3 Axisymmetric model of overmoulded resistor on

\section{RESULTS AND DISCUSSION}

\subsection{Two-dimensional Thermal Models}

To begin with, 2D axisymmetric models were created of simple test structures in order to carry out rapid analysis. An axisymmetric model of a single surface mounted chip resistor on an encapsulated PCB was constructed as shown in figure 3. Heat generated at the top face of the resistor was conducted through the resistor, substrate and the polymer before being dissipated to the ambient air (at $20^{\circ} \mathrm{C}$ ) by natural convection. The software used standard correlations to estimate the convective coefficients. The major assumptions in these models were that the polymer was in intimate contact with the resistor/substrate assembly and that there was no gap between the resistor and substrate. It was also assumed that heat was generated uniformly over the top face of the resistor. Figure 4 shows a predicted steady state contour profile for the structure at an input power of 0.25 Watt.

\subsubsection{Effect of Voids on Heat Dissipation}

These simple models were used to examine the effect that imperfections in the moulding process might have on the device performance. It is important that the moulding process is optimised so that large voids are avoided, especially as the presence of voids adjacent to heat generating components introduces a further barrier to the heat removal, potentially causing a significant increase in temperature rise of that component. This phenomenon was investigated using the axisymmetric model by introducing a layer of air adjacent to the top of the resistor to mimic a cavity. Two scenarios were considered: one where the void covered approximately $30 \%$ of the resistor area and one at $100 \%$. Figure 5 shows contour maps of steady state temperatures for the assembly without and with the $100 \%$ void. Table 2 summarises the maximum simulated temperature rise at $0.5 \mathrm{~W}$ and $1 \mathrm{~W}$ input powers. These results demonstrate that a void completely covering the top surface of a resistor can cause an increase of $17.5^{\circ} \mathrm{C}$ at $0.5 \mathrm{~W}$ and 35 ${ }^{\circ} \mathrm{C}$ at $1 \mathrm{~W}$ input over a void-free structure.

\section{FR4 substrate}

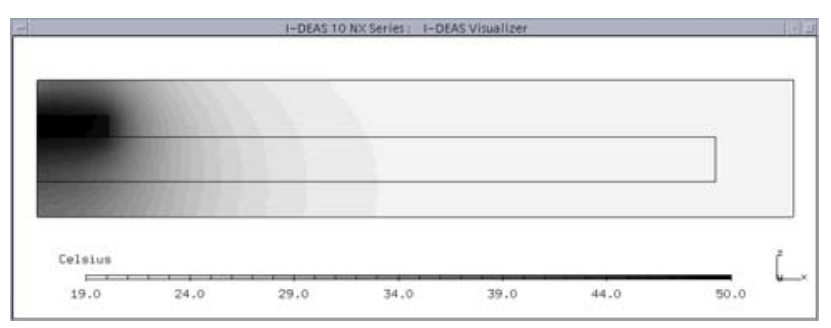

Fig. 4 A contour map showing the simulated steady state temperatures

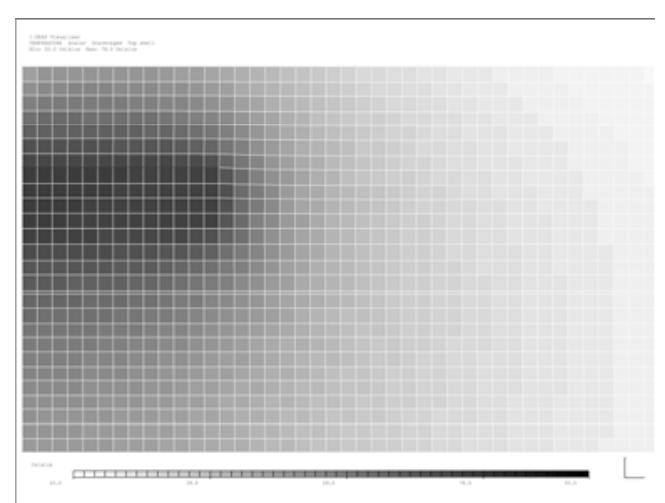

(a)

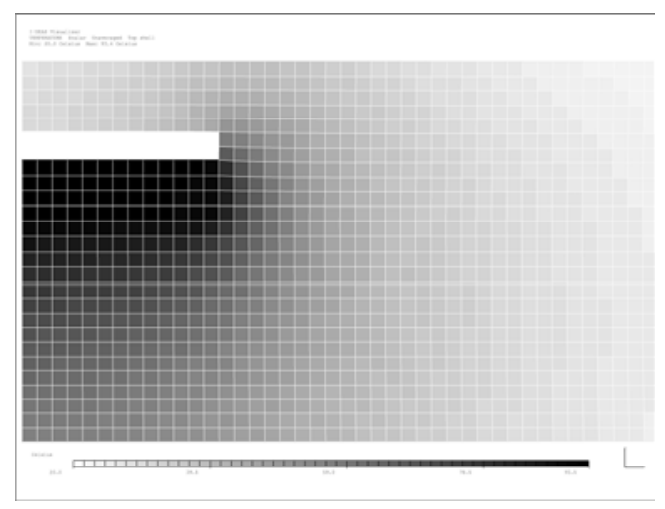

(b)

Fig. 5 Contour plot showing steady state temperatures (a) without and (b) with a void adjacent to a resistor 


\begin{tabular}{|c|c|c|c|}
\hline \multirow{2}{*}{$\begin{array}{c}\text { Input Power } \\
(W)\end{array}$} & \multicolumn{3}{|c|}{ Max Temperature Rise $^{\circ} \mathbf{C}$} \\
\cline { 2 - 4 } & No Void & $\mathbf{3 0 \%}$ coverage & $\mathbf{1 0 0 \%}$ coverage \\
\hline 0.5 & 55.9 & 58.13 & 73.4 \\
\hline 1 & 110.2 & 114.6 & 145.0 \\
\hline
\end{tabular}

Table 2 Effects of a void on temperatures

\subsection{Three-dimensional Models}

Following preliminary 2D analyses, more detailed three dimensional models of the test structures were prepared within the I-DEAS software package. The models described in this section were all constructed based on the geometry of figure 1 , with three 2512 resistors on a $1.6 \mathrm{~mm}$ thick FR4 substrate, which itself was centrally positioned in two layers of polymer overmould. The heat was assumed to be generated uniformly over the top surface of the chip resistors and, after being conducted through the different layers of the structure, was naturally convected to the surrounding ambient from all surfaces of the polymer encapsulation.

\subsubsection{Steady State Thermal Analysis}

Figure 6 illustrates four views of the model, (a) shows the overmoulded resistor/substrate combination and (b) the

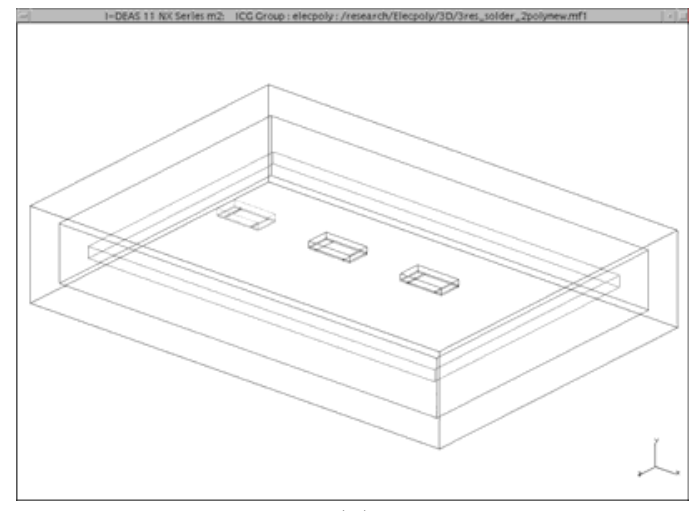

(a)

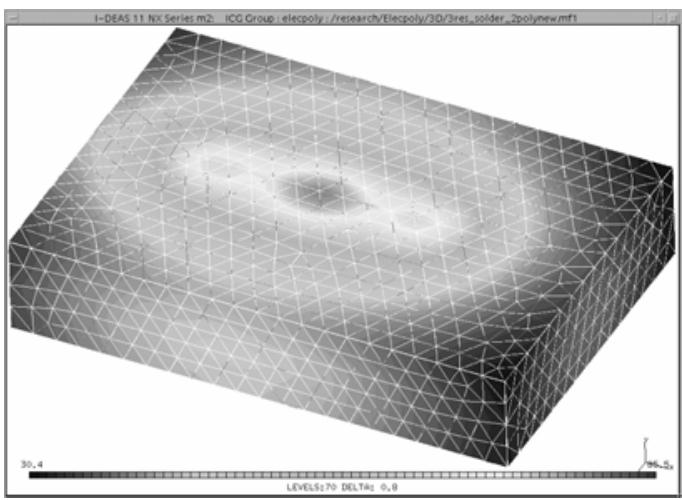

(c) surface mounted resistors on the FR4 substrate. (c) and (d) are steady state temperature contour maps for the top surface of the polymer and the resistor/FR4 respectively with an input power of $0.5 \mathrm{~W}$ per resistor. As expected the polymer overmould provided a significant thermally insulating layer that limited the dissipation of heat from the devices, such that a maximum temperature rise of $88.4{ }^{\circ} \mathrm{C}$ was recorded for the central resistor with the particular polymer encapsulation. For comparison, a model of the same structure without polymer overmoulding showed a temperature rise of only $74.7^{\circ} \mathrm{C}$.

\subsubsection{Transient Thermal Models}

In order to explore how the devices may perform when subjected to a transient power input, the 3D FE model with three 2512 SMT resistors was used in a transient thermal analysis. In the simulation, each resistor in the assembly was subjected to an instantaneous power input of $1 \mathrm{~W}$ and the resulting thermal effects were monitored for a period of 5 minutes. The simulation was also repeated for the same board assembly without polymer encapsulation, where the heat was convected directly to the ambient air. The temperature/time results of the simulations are given in figure 7 for the centre point of the top of the resistor mounted at the middle of the board.

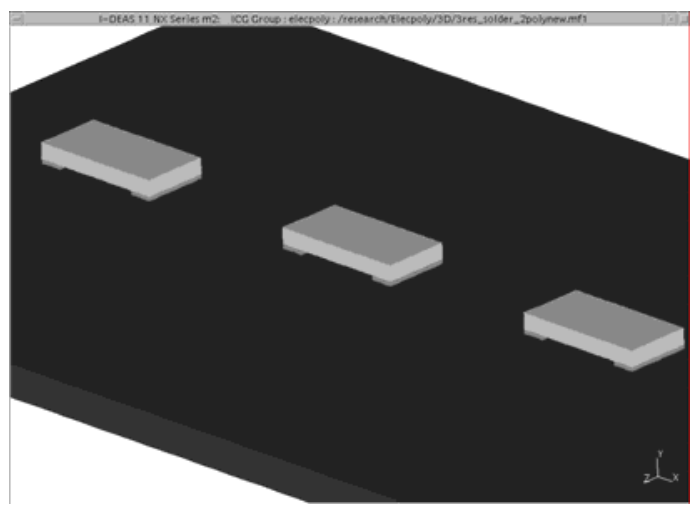

(b)

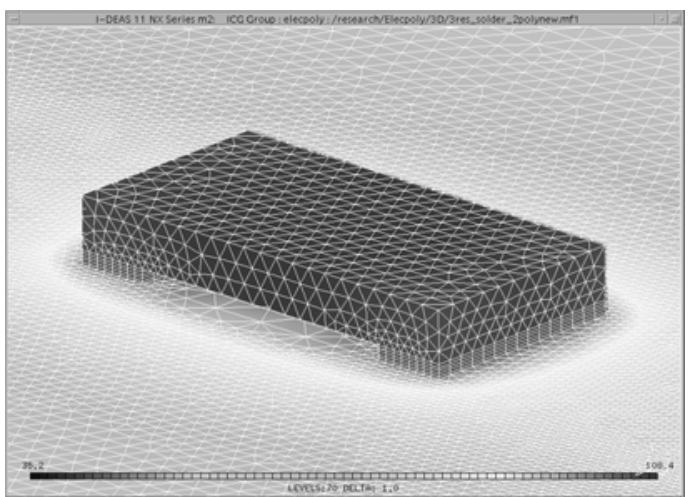

(d)

Fig. 6 3D Finite element model of overmoulded resistor/substrate 
The results demonstrated that the assembly without the polymer encapsulation heated up at a higher rate than the overmoulded assembly, which was slowed due to the extra thermal mass introduced by the polymer. However with prolonged power input, the bare device reached a steady state after around 100 seconds, while the polymer encapsulated board continued to heat up ultimately leading to a higher steady state temperature, in agreement with the steady state simulations, due to the effect of surrounding the electronics with a low thermal conductivity polymer.

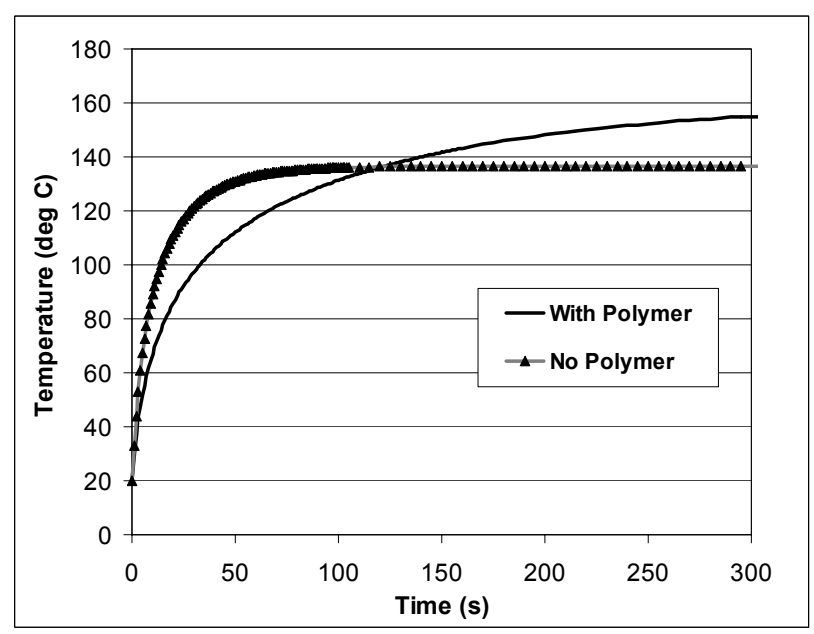

Fig. 7 Transient simulation results

\subsubsection{Effect of Filler Fraction on Thermal Performance}

Using the FE models developed it was possible to investigate how changing the material properties of the encapsulating polymers would influence the heat dissipation. This would assist in the selection of appropriate additives to the polymer and provide information on the expected gain to be made through variations in polymer formulation. In order to enhance the ability of the assembly to dissipate the heat generated by the circuit components, an investigation was carried out by the project partners to increase the thermal conductivity of the polymer by addition of a filler of relatively high thermal conductivity to the polymer blend.

To produce representative models of the devices the variation in thermal conductivity of the encapsulant as a function of filler content needed to be established. As relevant data for the materials in use was not available this had to be estimated based on published analytical models. A number of theoretical and empirical models for the prediction of the effective thermal conductivity of two phase mixtures were investigated by Tavman [3] and compared with experimentally measured data. Tavman found that a geometrical mean model provided the best estimation of the thermal conductivity of the resultant polymer at all filler percentages examined. The model is given below:

$$
K_{c}=K_{f}^{\phi} \cdot K_{m}^{(1-\phi)}
$$

Where $\phi$ is the volume fraction of the filler and $K_{c}, K_{f}$ and $K_{m}$ are the thermal conductivities of composite, filler and polymer respectively. Based on this equation, figure 8 illustrates the effect of the filler fraction within the polymer on the thermal conductivity for two different fillers namely: alumina and AlN. Conductivity values of $0.25 \mathrm{~W} / \mathrm{mK}, 25 \mathrm{~W} / \mathrm{mk}$ and $175 \mathrm{~W} / \mathrm{mK}$ were used for polymer, alumina and AIN respectively.

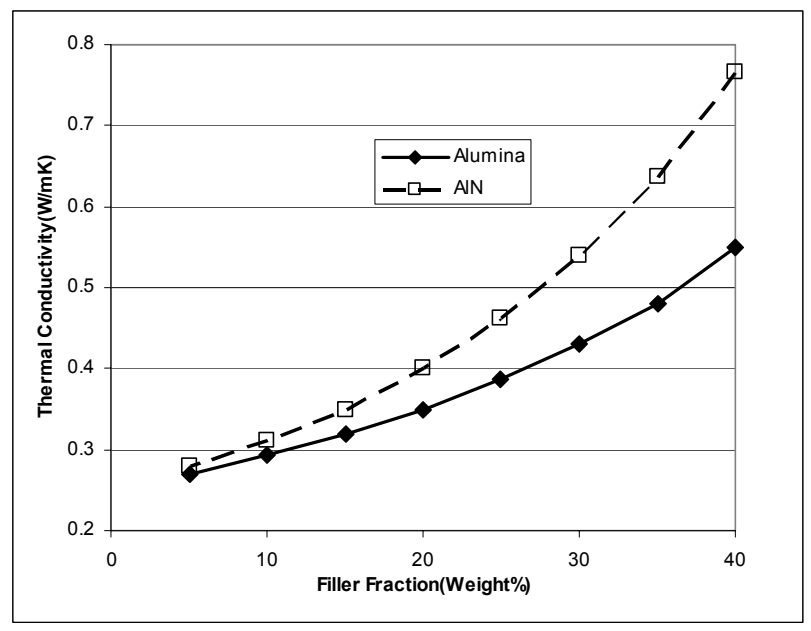

Fig. 8 Effect of filler fraction on thermal conductivity of polymer

Using the data presented above, a series of simulations were carried out to determine the effect of filler content within the inner soluble layer of polymer on the temperature rise sustained within the assembly when running at a power of $0.5 \mathrm{~W}$ per resistor. Figure 9 shows the results of these simulations and, as expected, demonstrates that introducing a filler with higher thermal conductivity can significantly influence the temperature rise experienced by the module.

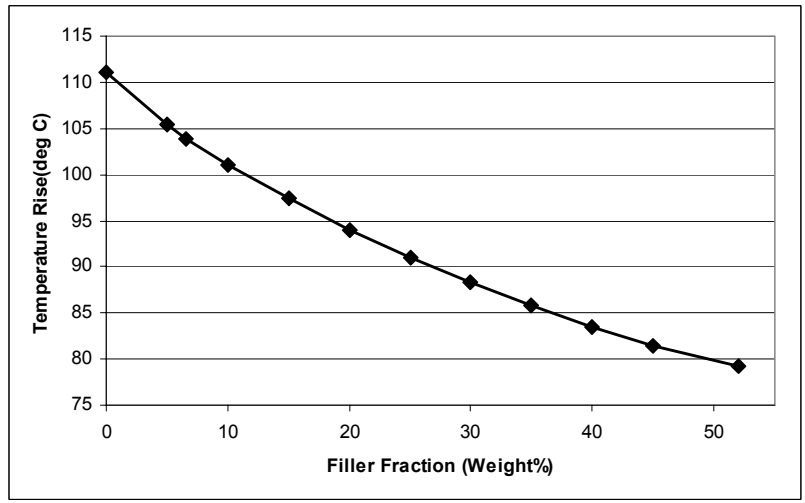

Fig. 9 Maximum steady state temperature rise within the assembly as a function of filler fraction for $\mathrm{Al}_{2} \mathrm{O}_{3}$ 


\subsubsection{Power De-rating}

From the results presented above, it is clear that the overmoulding of electronic circuits has an impact on the heat dissipation, which causes an increase in the temperatures experienced within the assembly. In order to avoid these temperature increases and achieve similar levels to circuits without any overmoulding, it would obviously be necessary to reduce the power levels in the assembly. To provide designers with simple rules it was necessary to determine the de-rating level required. A series of simulations were carried out using the same 3D model with 3 resistors and two layers of encapsulation. The material properties of the candidate polymer were used in this study. It was found that a drop in power of $16 \%$ was needed to reduce the maximum temperatures $\left(\mathrm{T}_{\max }\right)$ of the overmoulded assembly to that with no encapsulation.

\subsection{Thermo-mechanical Models}

For thermo-mechanical modelling, a 3D FE model was used, similar to that described for transient thermal modelling of the three 2512 SMT resistors mounted on a PCB. Initially, a thermal analysis was performed and the results were translated into an ABAQUS solver input file for subsequent analysis of displacements and stresses.

\subsubsection{Effect of Overmoulding on Thermo-mechanical Behaviour of Circuit Boards}

To begin with, an analysis was conducted to acquire an overall sense of the thermo-mechanical behaviour of an overmoulded circuit board compared to that without overmoulding. The performance of the circuit board was simulated assuming a uniform temperature increase of $100{ }^{\circ} \mathrm{C}$ (i.e. no power input to the device). Figure 10 shows contour plots of the maximum displacement results for both situations and are displayed with the same greyscale for direct comparison. The results clearly demonstrate an increase in the distortion levels in the encapsulated case - an approximately threefold rise in the maximum displacement is predicted. For the unencapsulated PCB a general bowing of the board can be seen which is attributed to the lower CTE of the ceramic components compared to the PCB material. For the encapsulated board there is less bowing which is likely to be due to the constraint of the overmoulding polymer. However, in this case, there is much more distortion of the board along its axis. This is thought to be due to the higher CTE of the overmoulding polymer compared to the FR4 which is placing the PCB in tension. In the current model it is assumed that there is perfect adhesion between the overmoulding and the PCB and therefore there is likely to be a significant shear stress at this interface. This highlights the importance of achieving good adhesion between the overmoulding polymer and the substrate. At present, this structure has not been modelled with low levels of adhesion at the interface, however it is anticipated that the mechanical interlocking of the polymer overmould with the protruding components is likely to lead to the development of shear stresses in the solder joints instead.

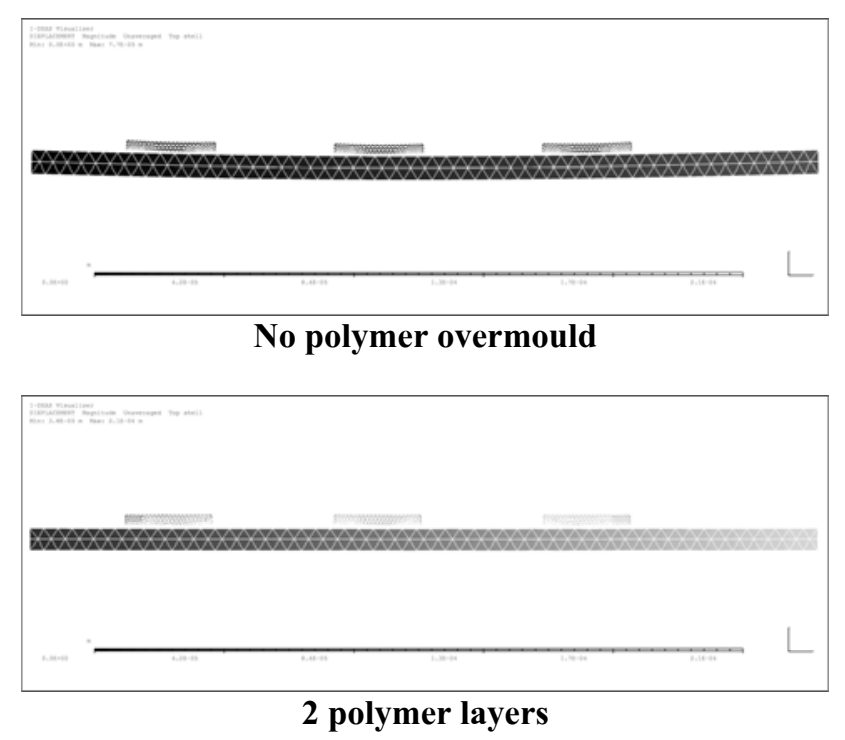

Fig. 10 Thermo-mechanical distortions for the resistors and substrate for a $100{ }^{\circ} \mathrm{C}$ uniform temperature rise

\subsubsection{Effect of Polymer Foaming on the Circuit Assembly}

As described earlier fillers were introduced to the polymer to increase the thermal conductivity of the first overmould shot in order to improve the heat dissipation from the circuit. A further investigation was carried out by the project partners to reduce the injection pressure during the overmoulding process in order to limit the induced stresses and distortions. If the stresses can be reduced at the overmoulding stage it is also expected that the in-service stresses will be reduced.

The injection pressure was reduced by the addition of blowing agent (BA) to the polymer mix resulting in a polymer foam. However, the foaming process in turn reduces the thermal conductivity of the polymer. Material properties for the recyclable polymer were measured using conventional techniques. Foamed polymer properties however had to be estimated using relationships given by Gibson and Ashby [4] for which the simplified equations are generally based on the relative density of foam/solid polymers. The coefficient of thermal expansion for most foamed polymers is similar to that of the solid that it is made of, while the Young's Modulus for a closed cell foam is a function of the density ratio and also fraction of the solid in the cell wall.

Thermal conductivity estimations based on these equations are summarised in table 3 showing, as expected, a reduction in thermal conductivity with an increase in the foaming level. 


\begin{tabular}{|l|c|c|}
\hline \multicolumn{1}{|c|}{ Material } & $\begin{array}{c}\text { Density } \\
\left(\mathbf{k g} / \mathbf{m}^{\mathbf{3}}\right)\end{array}$ & $\begin{array}{c}\text { Thermal Conductivity } \\
(\mathbf{W} / \mathbf{m K})\end{array}$ \\
\hline $\begin{array}{l}\mathrm{WSP}+30 \% \mathrm{Al}_{2} \mathrm{O}_{3} \\
(\text { Measured })\end{array}$ & 1520 & 0.43 \\
\hline $\begin{array}{l}\mathrm{WSP}+30 \% \mathrm{Al}_{2} \mathrm{O}_{3} \\
+1 \% \mathrm{BA}\end{array}$ & 1230 & 0.27 \\
\hline $\begin{array}{l}\mathrm{WSP}+30 \% \mathrm{Al}_{2} \mathrm{O}_{3} \\
+2 \% \mathrm{BA}\end{array}$ & 820 & 0.195 \\
\hline $\begin{array}{l}\mathrm{WSP}+30 \% \mathrm{Al}_{2} \mathrm{O}_{3} \\
+3 \% \mathrm{BA}\end{array}$ & 640 & 0.16 \\
\hline
\end{tabular}

Table 3 Density and thermal conductivity of candidate polymer at different foaming levels

Figure 11 is a graph showing the elastic modulus as a function of temperature for the solid polymer (as measured) and the estimated values for the foamed materials prepared with 1 to 3 percent of blowing agent.

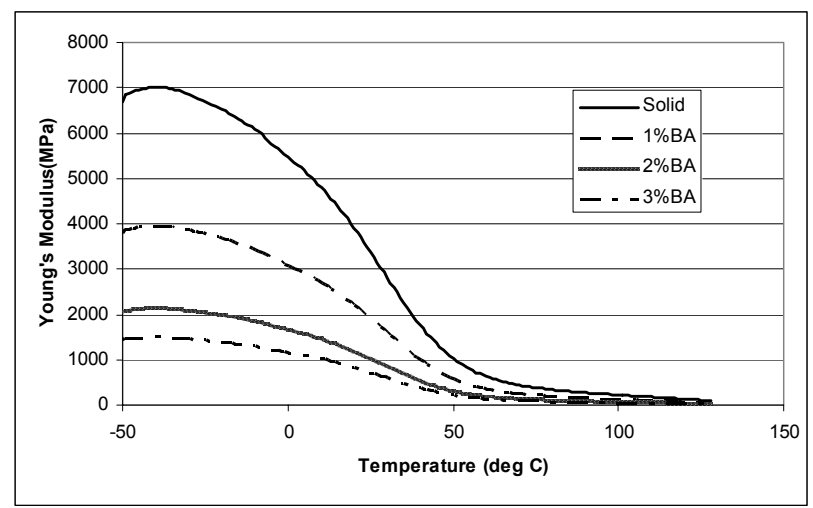

Fig. 11 Estimated elastic modulus for polymer at different foaming level

A simulation study was conducted to explore the effect of foaming on temperatures and stresses within the assembly. In the first instance, thermo-mechanical simulations were carried out using the ABAQUS software on the 3 resistor assembly, applying a uniform $100{ }^{\circ} \mathrm{C}$ temperature rise to the whole unit. Properties for the first polymer layer were adjusted in each simulation to reflect the measured/estimated values but the properties for other materials were fixed. The peak Von Mises stress values for the solid and foamed polymer with different percentage of blowing agent are given in table 4 . The results show significant reduction in the peak von Mises value within structures encapsulated with foamed polymers, a drop of $54.9 \%$ when foamed with $2 \%$ of blowing agent. This demonstrated that the process of foaming could have a significant effect on the overall stress levels within the assembly.

\begin{tabular}{|l|c|c|}
\hline Polymer Material & $\begin{array}{c}\text { Thermal Conductivity } \\
\text { (W/mK) }\end{array}$ & $\begin{array}{c}\text { Peak von } \\
\text { Mises (MPa) }\end{array}$ \\
\hline $\begin{array}{l}\text { Solid polymer } \\
\text { (Measured) }\end{array}$ & 0.43 & 464.3 \\
\hline $\begin{array}{l}\text { Foamed polymer } \\
\text { using 1\%BA } \\
\text { (estimated) }\end{array}$ & 0.27 & 318.9 \\
\hline $\begin{array}{l}\text { Foamed polymer } \\
\text { using 2\%BA } \\
\text { (estimated) }\end{array}$ & 0.195 & 209.3 \\
\hline
\end{tabular}

Table 4 Stress results simulated for the solid and foamed polymers after $100^{\circ} \mathrm{C}$ temperature rise

Further simulations were conducted to examine how the reduction in thermal conductivity would influence the heat dissipation and consequently the stress distribution. Analyses were carried out on the same assembly, applying $0.5 \mathrm{~W}$ of input power to each resistor. The results are summarised in table 5 for the solid polymer and that foamed with $2 \%$ blowing agent. As expected a larger temperature rise was observed with the foamed encapsulant, however despite this, the level of stress developed was still substantially lower. The tradeoff between temperature and stress should therefore be carefully considered when selecting the amount of blowing agent to use.

\begin{tabular}{|l|c|c||c|}
\hline $\begin{array}{c}\text { Polymer } \\
\text { Material }\end{array}$ & $\begin{array}{c}\text { Thermal } \\
\text { Conductivity } \\
(\mathbf{W} / \mathbf{m K})\end{array}$ & $\begin{array}{c}\text { Max } \\
\text { Temp } \\
\left({ }^{\circ} \mathbf{C}\right)\end{array}$ & $\begin{array}{c}\text { Peak von } \\
\text { Mises } \\
\text { (MPa) }\end{array}$ \\
\hline $\begin{array}{l}\text { Solid polymer } \\
\text { (Measured) }\end{array}$ & 0.43 & 108.4 & 291.0 \\
\hline $\begin{array}{l}\text { Foamed polymer } \\
\text { using 2\%BA }\end{array}$ & 0.195 & 141.8 & 180.9 \\
\hline
\end{tabular}

Table 5 Simulated temperature and stress results for the solid and foamed polymer

\section{CONCLUSIONS}

Finite element models have been used to carry out design studies on polymer encapsulated electronics packages. 2D axisymmetric models have been used to predict the thermal behaviour of assemblies in simpler analyses and where more detailed representation of the circuit layout has been required for complex thermal and thermomechanical analysis, 3D models have been developed.

As expected, encapsulating the electronics in a polymer limits the heat loss from the electronics due to their inherent poor thermal conductivity. As part of this work, various methods to dissipate the heat more efficiently have been evaluated, using the thermal models as a means. A method of increasing the thermal dissipation from the assembly has been studied whereby particles of higher conductivity are added to the polymer. 
Results have been presented of the increase in thermal conductivity of polymer as a function of the filler fraction for two different filler materials showing the improvement that can be gained in thermal dissipation. However, despite this, it has been demonstrated that a $16 \%$ de-rating of power would be required for the particular two-shot overmould to achieve temperatures similar to that obtained without any polymer encapsulation.

Through the application of 2D axisymmetric models it was possible to demonstrate that voids in the polymer encapsulant adjacent to a heat generating circuit component can result in considerable increase in temperatures of that component. These results have indicated that it is necessary to optimise the polymer formulation and the overmoulding process to improve adhesion between the polymer and the circuit board and to minimise the possibilities of cavities forming within the structure.

By using a foaming process, reductions in the moulding injection pressures can be achieved and thermo-mechanical models have shown that this reduces stress in the circuit board although it has the undesirable effect of reducing the thermal conductivity of the polymer. Further work needs to be carried out to understand the importance of the adhesion of the polymer to the $\mathrm{PCB} /$ components and to investigate alternative ways to conduct heat from the assembly to improve the thermal performance. In addition, more detailed thermomechanical studies and validation are required to examine the reliability of such assemblies operating in harsh environments.

\section{ACKNOWLEDGMENTS}

The authors acknowledge the financial support of the UK Department of Trade and Industry (DTI) under the Foresight Vehicle LINK programme and the technical support of TRW Limited, PERA International, PVAXX Research and Development Limited, Demag Hamilton Limited and Rosti.

\section{REFERENCES}

[1] Directive 2000/53/EC of the European Parliament and of the Council of 18 September 2000 on end-oflife vehicles, Official Journal of the European Communities, 21 October 2000.

[2] F. Sarvar, N.J. Teh, D.C. Whalley, D.A. Hutt and P.J. Palmer, "Thermo-mechanical Modeling of Polymer Encapsulated Electronics" , Proceedings of the 9th IEEE I-THERM Conference, Las Vegas, USA, May 2004, pp 465-472, ISBN 0-7803-8357-5.

[3] H. Tavman, "Thermal Conductivity of Particle Reinforced Polymer Composites", NATO-Advance Study Institute (ASI) Nanoengineered Nanofibrous
Materials Conf., September 1-12, 2003, Antalya, Turkey, pp 449-455.

[4] L. J. Gibson and M. F. Ashby, Cellular Solids Structure and Properties, Second Edition, Cambridge University press, 1997. 